\title{
Can Normal Fracture Healing Be Achieved When the Implant Is Retained on the Basis of Infection? An Experimental Animal Model
}

\author{
Fuat Bilgili MD $\mathbb{1}$, Halil Ibrahim Balci MD, Kayahan Karaytug MD, \\ Kerim Sariyilmaz MD, Ata Can Atalar MD, Ergun Bozdag PhD, \\ Meral Tuna PhD, Bilge Bilgic MD, Nezahat Gurler MD
}

Published online: 16 May 2015

(C) The Association of Bone and Joint Surgeons (B) 2015

\begin{abstract}
Background Infection after open fractures is a common complication. Treatment options for infections developed after intramedullary nailing surgery remain a topic of controversy. We therefore used a rat fracture model to evaluate the effects of infection on osseous union when the implant was maintained.
\end{abstract}

Each author certifies that he or she, or a member of his or her immediate family, has no funding or commercial associations (eg, consultancies, stock ownership, equity interest, patent/licensing arrangements, etc) that might pose a conflict of interest in connection with the submitted article.

All ICMJE Conflict of Interest Forms for authors and Clinical Orthopaedics and Related Research ${ }^{(\mathbb{R}}$ editors and board members are on file with the publication and can be viewed on request.

Clinical Orthopaedics and Related Research ${ }^{\mathbb{B}}$ neither advocates nor endorses the use of any treatment, drug, or device. Readers are encouraged to always seek additional information, including FDAapproval status, of any drug or device prior to clinical use.

Each author certifies that his or her institution approved the animal protocol for this investigation and that all investigations were conducted in conformity with ethical principles of research. The study design, animal surgeries, statistical analysis, and manuscript writing were performed in the Department of Orthopedics and Traumatology, Istanbul Faculty of Medicine, Istanbul University, Istanbul, Turkey. Mechanical testing was performed in the Laboratory of Biomechanics and Strength of Materials, Faculty of Mechanical Engineering, Istanbul, Technical University, Istanbul, Turkey. Histological evaluation was performed in the Department of Pathology, Istanbul Faculty of Medicine, Istanbul University, Istanbul, Turkey. Bacterial isolation was performed in the Department of Microbiology, Istanbul Faculty of Medicine, Istanbul University, Istanbul, Turkey.

F. Bilgili ( $₫$ ), H. I. Balci, K. Karaytug, A. C. Atalar Department of Orthopedics and Traumatology, Istanbul Faculty of Medicine, Istanbul University, Fatih/Capa, Istanbul 34093, Turkey

e-mail: fuatbilgili@gmail.com
Questions/purposes In a rat model, (1) does infection alter callus strength; (2) does infection alter the radiographic appearance of callus; and (3) does infection alter the histological properties of callus?

Methods An open femoral fracture was created and fixed with an intramedullary Kirschner wire in 72 adult male Sprague-Dawley rats, which were divided into two study groups. In the infection group, the fracture site was contaminated with Staphylococcus aureus (36 animals), whereas in the control group, there was no bacterial contamination (36 animals). No antibiotics were used either for prophylaxis or for treatment. We performed biomechanical (maximum torque causing failure and stiffness), radiographic (Lane and Sandhu scoring for callus formation), and histologic (scoring for callus maturity) assessments at 3 and 6 weeks. The number of bacteria colonies on the femur, wire, and soft tissue inside knee were compared to validate that we successfully created an infection model. The number of bacteria colonies in the soft tissue inside the knee was higher in the infection group after 6 weeks than after the third week, demonstrating the presence of locally aggressive infection.

Results Infection decreased callus strength at 6 weeks. Torque to failure $(299.07 \pm 65.53 \mathrm{Nmm}$ versus $107.20 \pm$ 88.81, mean difference with $95 \%$ confidence interval, 192 [43-340]; $\mathrm{p}=0.007)$ and stiffness at 6 weeks $(11.28 \pm 2.67$ $\mathrm{Nmm}$ versus $2.03 \pm 1.68$, mean difference with $95 \%$ confidence interval, 9 [3-16]; $p=0.004$ ) both were greater in the control group than in the group with infection.

\footnotetext{
K. Sariyilmaz

Department of Orthopedics and Traumatology, Acibadem Atakent University Hospital, Istanbul, Turkey
} 
Radiographic analysis at 6 weeks demonstrated the fracture line was less distinct (Lane and Sandhu score of 2-3) in the infection group and complete union was observed (Lane and Sandhu score of 3-4) in the control group ( $p=0.001)$. Semiquantitative histology scores were not different between the noninfected controls and the rats with infection (score 10 versus 9).

Conclusions Retaining an implant in the presence of an underlying infection without antibiotic treatment leads to weaker callus and impedes callus maturation compared with noninfected controls in a rat model. Future studies might evaluate whether antibiotic treatment would modify this result.

Clinical Relevance This model sets the stage for further investigations that might study the influence of different interventions on fracture healing in implant-associated osteomyelitis. Future observational studies might also evaluate the histological properties of callus in patients with osteomyelitis.

\section{Introduction}

Infection after open reduction and internal fixation is common, and its treatment remains a topic of debate. Maintaining bony stability is essential until fracture union is complete, but the surgery itself and internal fixation hardware both can potentiate infection because of tissue devitalization and colonization of the hardware $[1,7,21]$. On the other hand, evidence also shows that the stability provided by retaining the implant reduces the likelihood of infection after fixation [19, 25, 27], and so controversies abound on this topic.

When infection develops after plate-screw fixation, retention of the implant until union is recommended if the fracture fixation is stable [5, 23, 27]. The treatments for infection after intramedullary nailing are difficult and more controversial. Commonly, the recommendation is for nail removal and débridement [26]. There is insufficient evidence as to whether the nail should be retained until union of the fracture area or removed immediately to begin

\section{E. Bozdag, M. Tuna}

Laboratory of Biomechanics and Strength of Materials, Faculty of Mechanical Engineering, Istanbul Technical University, Istanbul, Turkey

B. Bilgic

Department of Pathology, Istanbul Faculty of Medicine, Istanbul University, Istanbul, Turkey

N. Gurler

Department of Microbiology, Istanbul Faculty of Medicine, Istanbul University, Istanbul, Turkey infection treatment. We sought to evaluate this question using a rat fracture model, specifically to evaluate the effects of infection on osseous union with maintenance of the implant.

We asked the following questions: (1) does infection alter callus strength; (2) does infection alter the radiographic appearance of callus; and (3) does infection alter the histological properties of callus?

\section{Material and Methods}

\section{Study Design}

This experimental study included a total of 72 male Sprague-Dawley rats (Istanbul University, Institute of Experimental Medicine) with an age of 14 weeks and a mean weight of 250 to $300 \mathrm{~g}$. Institutional Animal Care and Use Committee approval was obtained before study initiation (ethics committee permission number 2012/161). The rats were randomly divided into two equal groups: the infection group (36 rats) and the control group (36 rats). All rats received an open fracture of the femoral diaphysis that was fixed with intramedullary Kirschner wire.

For surgical anesthesia, all the animals were administered an intraperitoneal injection of $65 \mathrm{mg} / \mathrm{kg}$ ketamine and $7 \mathrm{mg} / \mathrm{kg}$ xylazine. Under sterile conditions, and through a lateral approach, we approached the middiaphysis of the femur, one-third of the diaphyseal diameter was drilled, and the remaining bone was broken manually. The knee was opened with a $1-\mathrm{cm}$ medial parapatellar incision, and the patella was dislocated laterally. After reduction of the fracture without damaging the periosteum, a 21-gauge needle was inserted in the intramedullary canal through the femoral intercondylar notch, and the femur was drilled to provide the most stable fixation possible, Kirschner wire (0.71-1.25 $\mathrm{mm}$ diameter) was advanced proximally to the greater trochanter, and the distal end of the wire was cut and positioned inside the intercondylar notch.

In the rats assigned to the infection group, we induced infection according to an established model [12] with methicillin-sensitive Staphylococcus aureus isolated from a patient with an intraarticular infection. For the inoculation procedure, the bacteria produced from fresh 24-hour cultures in bloody agar were prepared as opaque suspensions in phosphate-buffered saline (PBS; $50 \mathrm{mM} \mathrm{NaH2PO} 4+$ $\mathrm{Na} 2 \mathrm{HPO} 4, \mathrm{pH} 7.2)$ in a $0.5 \mathrm{McF}$ arland tube $\left(1 \times 10^{8}\right.$ colony-forming units $[\mathrm{CFU}] / \mathrm{mL})$. The suspensions were used to create the infection model in half of the rats. For postoperative pain control, the rats were administered 0.2 $\mathrm{mg} / \mathrm{kg}$ morphine sulfate subcutaneously immediately after surgery, twice in the first 24 postoperative hours, and when 
necessary for sufficient analgesia. The animals were evaluated twice a day for swelling, complications, nutrition, and hydration. Postoperatively, the rats were fed a standard diet and water. At the end of the study period, all animals were euthanized with an intraperitoneal injection of $135 \mathrm{mg} / \mathrm{kg}$ sodium pentothal. Aseptic techniques were used throughout all the procedures.

In the control group, $10 \mu \mathrm{L}$ PBS (control) was injected into the fracture area after fixation of the fracture created using the same technique. The incisions in both study groups were closed with 5-0 Vicryl sutures and skin staples.

The rats in each group (36 rats) were subdivided into groups of 18 and follow-up examinations were completed at 3 and 6 weeks after surgery. At each time point, eight rats were used for biomechanical examination, eight rats were used for microbiological investigation, and two rats were used for histological examination.

To validate the presence of systemic infection, blood samples from the infection group were taken from large vessels after euthanasia. Blood samples (approximately 2.0 $\mathrm{mL}$ ) were collected aseptically into blood culture tubes and placed in a BACTEC 9120 device (Becton Dickinson Microbiology Systems, Franklin Lakes, NJ, USA) in Istanbul University, Department of Microbiology. After reproduction in the tubes, implantation was made from each tube into a bloody gelatinous medium. After 24 to 48 hours of incubation at $37{ }^{\circ} \mathrm{C}$, the bacteria produced were identified. Methicillin-sensitive $S$ aureus was produced from the blood samples in the infection group.

Quantitative bacteriology assessments on the femur, wire, and soft tissue inside the knee were performed by one observer (NG) and the groups were compared to validate that we successfully created an infection model (Table 1).

After euthanasia, synovial samples were taken for aerobic culture from the joints of both the operated and non-operated sides from all of the animals. After 48 hours of incubation at $37{ }^{\circ} \mathrm{C}$, positive or negative changes were recorded, and it was noted if the blood culture had become opaque. Samples were obtained aseptically from both femurs, and culture and bacteria counts were completed.

The wires were removed aseptically from the operated femurs. Under sterile conditions, the bacteria attached to the wire were separated by centrifugation in a sonication device $(67 \mathrm{kHz}$ at $11,000 \mathrm{rpm})$. For microtiter dilution, the samples were collected, and the bacteria count (CFU/wire) was calculated for each wire. No bacterial colonization occurred in the femurs of the control group. Based on colony counts, as expected, the infections worsened over the period of observation such that the number of bacterial colonies in the soft tissue around the knee was higher in the infected rats after 6 weeks than at the 3-week time point (338 at 6 weeks, seven at 3 weeks; $p=0.002$ ) (Table 1).

Using a digital imaging system, radiographs were taken for all rats at 3 and 6 weeks after surgery. The radiographs were taken under anesthesia with the rats in the prone position and both legs abducted. The callus tissue and osseous union were evaluated according to the Lane and Sandhu system, which scores fracture healing as 0 (no callus), 1 (callus formation present but no evidence of bone union), 2 (initiation of bone union present), 3 (bone union present, fracture line no longer visible), or 4 (complete bony union) [14].

For histological evaluation, after implants were removed, the femurs were removed and fixed in $10 \%$ formalin for 1 day and then decalcified in a $10 \%$ nitric acid solution for 2 days. The tissue samples were embedded in paraffin after tissue processing. The sections were stained with hematoxylin-eosin and examined histopathologically for inflammation and callus formation using a scoring system [12] that describes the callus formation from fibrous tissue to bone healing with matured bone.

A total of 32 animals underwent biomechanical evaluation. After euthanasia, both femora were removed by disarticulation of the hip and knee. The intramedullary wires were removed by grasping the end of each wire as it protruded from the distal part of the femur, and soft tissue was dissected from the bone except for a thin layer covering the callus. The rat femur samples were first fixed from both ends with paste (steel paste-polyester resin) inside cylindrical aluminum pipes. To prevent the bones from turning, the pipes were fixed to metal cylinder blocks. The upper part of the bone fixed on the metal blocks was attached to the upper clamp of a universal test machine (MTS 858 Mini Bionix II, Eden, Prairie, MN, USA), and the lower part of the bone was attached to the lower clamp. The bones were attached to the load cell to measure the axial and torsional loads (MTS Axial-Torsional Load Transducer [2500 N/25 Nm]).

After attaching the samples to the test machine, each sample was exposed to torsional loading at $90 \% \mathrm{~min}$ angular

Table 1. Number of bacteria produced in the femur, wire, and soft tissues of the infection group (CFU)

\begin{tabular}{lllr}
\hline Postoperative period & Femur (CFU) & Wire (CFU) & Soft tissue in the knee (CFU) \\
\hline Third week & $431 \pm 377(70-1000)$ & $166 \pm 147(16-400)$ & $7 \pm 14(0-40)$ \\
Sixth week & $650 \pm 327(300-1000)$ & $586 \pm 421(120-1000)$ & $338 \pm 280(50-800)$ \\
\hline
\end{tabular}

Values are mean \pm SD with ranges in parentheses; $\mathrm{CFU}=$ colony-forming units. 
speed. The tests continued until failure was observed. By applying the same test scenario to each sample, the time, torsion angle, and torsion moment values were recorded simultaneously at a $10-\mathrm{Hz}$ frequency.

All the collected data were processed in MATLAB (Matworks, Inc, Natick, MA, USA) and the values for maximum moment load (Nmm) leading to failure during loading and the torsional angle (degree) at the moment of damage for each sample were obtained. The rigidity was calculated from the measured torsional angle and moment values. The maximum torque values were normalized by the respective values from the intact femurs (percent maximum torque) and average values were calculated for each group.

One rat died from the infection group of rats assigned to the biomechanical study. In the control group, two rats were withdrawn from the study on the suspicion of contamination. Thus, three rats were added to the study so as not to reduce the power of the study.

The structural differences between the animals were minimized by calculating the maximum torque causing failure (percent torque) in the contralateral healthy femur.

\section{Statistical Analysis}

A power analysis based on a previous study was used to determine the number of animals [2]. Assuming a power level of 0.8 and $p$ value of 0.05 , six animals would be required to determine a $60 \%$ difference between infection and noninfection groups in terms of mechanical testing. We choose eight rats per group to increase the power level and consequently our power level increased to 0.9 .
A two-way variance analysis was used for the biomechanical values. The two factors were time and fracture model. A value of $p<0.05$ was accepted as statistically significant. Differences between fracture types over time were assessed using the Bonferroni-Dunn post hoc test when appropriate. Statistical analysis was performed using SPSS software (IBM, Chicago, IL, USA).

\section{Results}

Infection decreased callus strength at 6 weeks but not at 3 weeks. Torque to failure and stiffness both were lower in the infection group than in the control group at 6 weeks, but with the numbers available for testing, they were not different at 3 weeks ( $\mathrm{p}=0.615$ and $\mathrm{p}=1$, respectively; Table 2 ). Torque to failure (299.07 $\pm 65.53 \mathrm{Nmm}$ versus $107.20 \pm 88.81$, mean difference with $95 \%$ confidence interval $[\mathrm{CI}]=192[43-$ 340]; $\mathrm{p}=0.007)$ and stiffness at 6 weeks $(11.28 \pm 2.67 \mathrm{Nmm}$ versus $2.03 \pm 1.68$, mean difference with $\mathrm{CI}=9$ [3-16]; $\mathrm{p}=$ 0.004 ) both were greater in the control group than in the group with infection. Maximum torque to failure was lower in the infection group than in the control group at all time points. Regarding biomechanical strength at 3 weeks postoperatively, there was a difference between the control and infection groups ( $41 \%$ versus $16 \%$ of contralateral femur, respectively; $p=0.019$ ). At 6 weeks, callus strength in the control group remained higher than in the infection group (62\% versus $29 \%$ of contralateral femur, respectively; $\mathrm{p}=$ 0.036) (Table 3).

Infection altered the radiographic appearance of callus. The radiographs taken at the third week revealed callus

Table 2. The maximum torque causing failure (Nmm) and stiffness ( $\mathrm{Nmm} /$ degree) of the groups after 3 and 6 weeks

\begin{tabular}{lccccc}
\hline Postoperative period & Infection group & Control group & p value & Infection group healthy side & Control group healthy side \\
\hline Maximum torque causing failure (Nmm) & & & & \\
$\quad$ Third week & $48.4 \pm 27.4$ & $116.3 \pm 44.2$ & 0.615 & $346.6 \pm 81.1$ & $332.5 \pm 103$ \\
$\quad$ Sixth week & $107.2 \pm 88.8$ & $299.1 \pm 65.5$ & 0.007 & $250.6 \pm 93$ & $410.5 \pm 104.4$ \\
Stiffness (Nmm/degree) & & & & \\
Third week & $0.9 \pm 0.6$ & $2.6 \pm 1.6$ & 1.000 & $20.1 \pm 7.1$ & $20.8 \pm 7.1$ \\
Sixth week & $2 \pm 1.7$ & $11.3 \pm 2.7$ & 0.004 & $14.1 \pm 4.7$ & $24.1 \pm 6$ \\
\hline
\end{tabular}

Values are mean $\pm \mathrm{SD}$.

Table 3. The maximum torque causing failure (Nmm) ratio of the groups after 3 and 6 weeks

\begin{tabular}{lcll}
\hline Postoperative period & Infection group & Control group & p value \\
\hline Maximum torque causing failure (Nmm) & & & \\
Third week & $16 \pm 11.1$ & $40.6 \pm 21.3$ & 0.019 \\
Sixth week & $28.8 \pm 24.6$ & $62.2 \pm 27$ & 0.036 \\
\hline
\end{tabular}

Values are mean $\pm \mathrm{SD}$. 

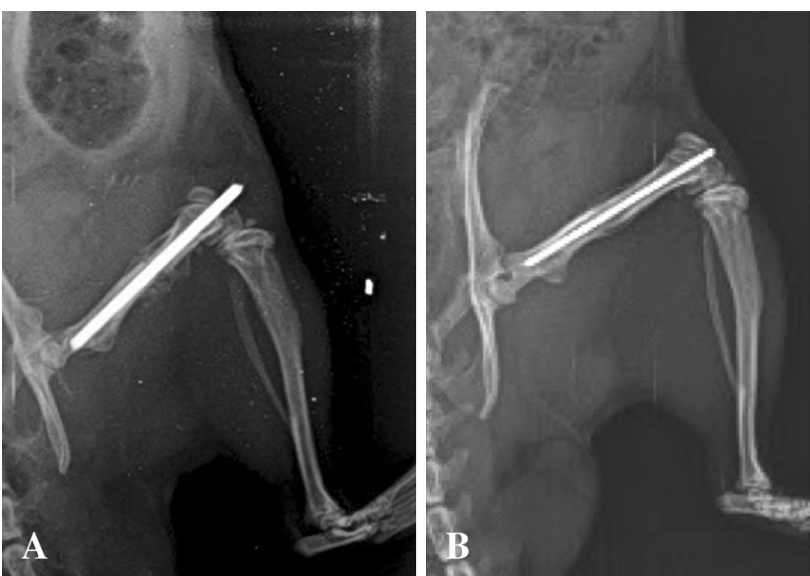

Fig. 1A-B Radiologic images at 6 weeks postoperatively are shown. Gross evaluation of radiographic image in the infection group reveals immature callus surrounding the fracture site and lack of bridging bone (A). Interval cortical bridging is seen in the control group (B).

formation in both groups, but no bony union could be detected. At the sixth week, complete bony union was found in the control group, whereas the infection group showed only the initial stages of bony union (Fig. 1). Three weeks after surgery, radiological evidence of fracture healing was seen both in the infection and the control groups (Lane and Sandhu score of 1-2). At 6 week, the fracture line was less distinct (Lane and Sandhu score of 2-3) in the infection group and complete union was observed (Lane and Sandhu score of 3-4) in the control group ( $\mathrm{p}=0.001)$.

Infection altered the histological properties of callus. Histological evidence of bone healing was observed by the sixth week in the control group but not in the infection group. There were areas concentrated with polymorphonuclear leukocytes (PMNs) and callus in all samples. Bony fragments were observed on careful examination of these PMNs, which function as a type of macrophage. In the third-week samples, there were cartilage areas (score 8: mostly immature bone and little cartilage tissue) in both groups. At the sixth week, the PMNs were denser and there were areas of abscess in the infection group (score 9: fracture healing with immature bone) (Fig. 2). In the control group, nearly mature bone tissue that showed trabecular formation was observed (score 10: fracture healing with mature bone) (Fig. 3).

\section{Discussion}

Infection after internal fixation is one of the most serious complications that can arise during fracture treatment. The metallic devices increase the likelihood of infection by providing a base for the formation of a biofilm layer; however, these osteosynthesis devices provide vital stability for

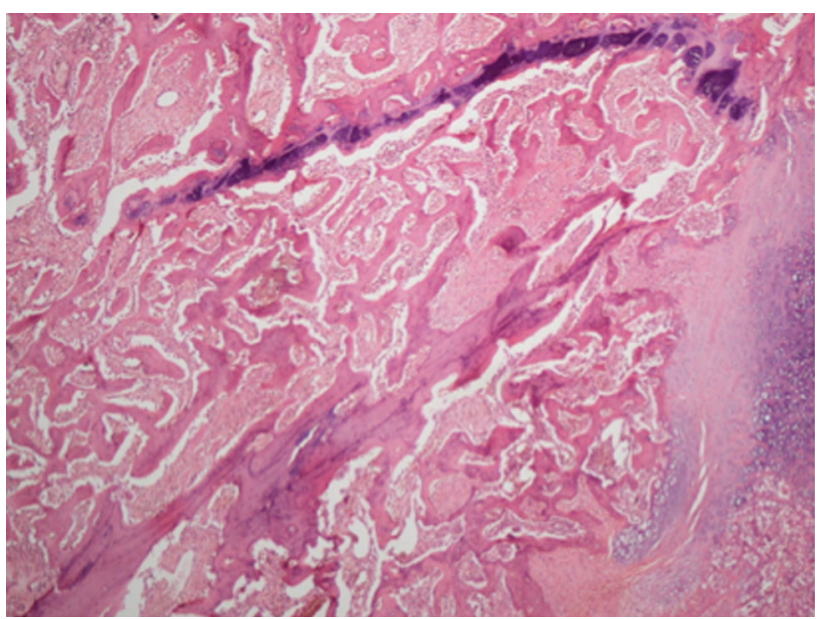

Fig. 2 The infection group at 6 weeks shows callus tissue, consisting predominantly of reactive trabecular bone and focally of cartilage (Stain, hematoxylin-eosin; original magnification, $\times 100$ ).

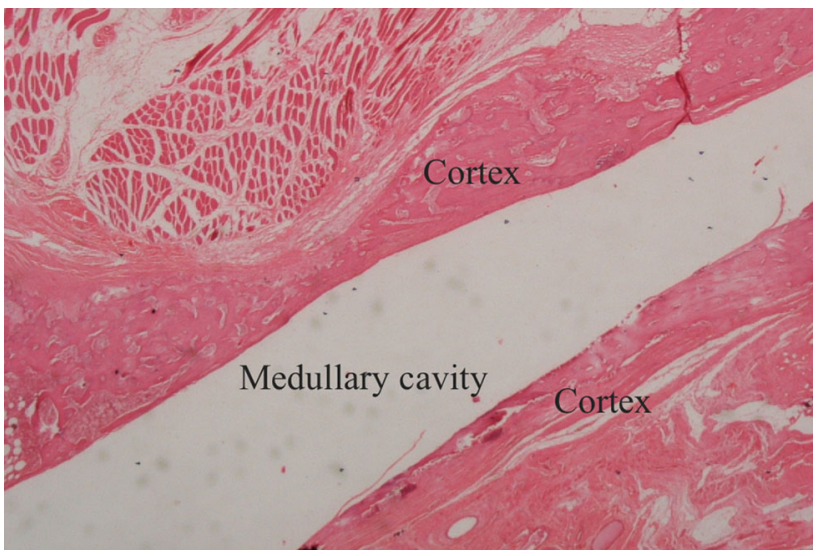

Fig. 3 Control group at 6 weeks shows callus tissue, reactive bone, showing bridging in the cortical area (Stain, hematoxylin-eosin; original magnification, $\times 40$ ).

fracture union $[10,18,20]$. Because of these competing concerns, two possible approaches can be considered when an infection arises after open reduction and internal fixation: implant removal and treatment of the infection or implant retention until fracture union with subsequent treatment and perhaps delayed implant removal. There are conflicting recommendations regarding this dilemma [1]. In this animal study we asked the question of whether infection alters fracture callus strength, formation, and morphology. Our findings showed that retaining an implant in the presence of an underlying infection (in the absence of antibiotic treatment) leads to weaker callus and impedes callus maturation compared with controls.

There are several limitations of this study. First, as noted, we did not give any antibiotics to the rats. In clinical 
practice, antibiotics are given according to the bacteria isolated from the infection side [3]. It is possible that the differences between the groups would have been less dramatic had we given antibiotics; however, to ensure infection in the infection group in this model, antibiotics were not used. Second, we euthanized the animals at the end of 6 weeks. Longer durations of followup may have resulted in stronger, more visible callus. However, 6 weeks was used because 6 weeks was the endpoint recommended in previous animal research [11]. In addition, the distal and proximal ends of the implant were not locked, which could lead to weaker torsional stability. The other limitation of this study was the absence of a control group treated without stabilization. Lastly, we examined only two rats in each group for histological comparison, and so if there were to have been animal-to-animal variability, we might have failed to discern it.

Although callus tissue formed, it was biomechanically weak. By 6 weeks, the infection group had lower failure torque and stiffness. This is the first study to our knowledge that compares the strength of the infected and noninfected callus. Fracture stability after osteosynthesis is important for fracture healing and preventing infection [25]. In previous animal studies, lower rates of infections were observed in fractures with stable fixation than in those with unstable fractures [19, 27]. In the current study, loosening around the implant was observed in the infection group. Fracture stability is also related to the biomechanics of the newly formed callus. In a previous animal study, the rigidity of osteotomies with unstable fixation was observed to be weak; however, no difference was observed between the bending rigidity of stable osteotomies and healthy bones [22].

The radiographs revealed that the callus tissue was not fully ossified, and a radiolucent area had formed around the implant in the infection group. In previous studies of infected fracture models, callus tissue was observed in the infected fracture area under the supply of antibiotics [2, 3, 24]. The difference from these studies is that no antibiotics were used and no segmental defects were created. Further work will be necessary to better understand the systemic antibiotic effect on controlling the infection and enhancing bone formation in our infection model.

In histological evaluation, the results were consistent, although two specimens in each group were evaluated [15]. The callus tissues of both groups at the third week were similar, but at the sixth week, the callus formation remained immature in the infection group. Larger sample sizes are needed for additional semiquantitative information.

The indications for the removal or retention of implants in cases of infection in the fracture area during the early period after osteosynthesis are not well defined. A retrospective clinical study examined the union for cases in which the implant was not removed after an early-stage infection in the fracture area [1]. The authors concluded that infection can be successfully treated with débridement and antibiotics with hardware retention. In addition, open fractures and intramedullary nailing were two poor prognostic factors associated with lower success rates [1]. The results of the current study tend to support these clinical study data with some limitations (lack of any treatment in the groups). Based on these two poor prognostic factors (open fractures and an intramedullary implant), complete union of the fracture was not observed.

An important part of this type of experimental study is the formation of the fracture and infection model. Rat osteosynthesis models have previously used open incisions and stabilization with plates or external fixator systems [4, $8,9,17]$. Although these systems are commonly associated with gap osteotomy models, a fracture model could be similarly achieved by making a single saw cut (rather than two needed for a gap osteotomy). In the current study, a transverse open fracture was created in the femoral middiaphyseal area to obtain a standard fracture model in the same area of the femur. The standard treatment for fractures in this area is fixation with intramedullary nailing. Accordingly, we placed a Kirschner wire inside the medullary canal for fixation and stabilization. $S$ aureus is the common cause of periprosthetic infections and is typically used to create an infection model [6, 19, 24, 25, 27]. Infection models have been created using a range of $S$ aureus concentrations such as $1 \times 10^{8}$ to $1 \times 10^{4}$ $[13,25]$. Clinically, the development of infection after intramedullary nailing may progress as far as sepsis. Even in the event of sepsis, it has been reported that union can still occur in a fracture that has stable fixation with intramedullary nailing [16]. In the current study, a high number of $S$ aureus $\left(1 \times 10^{8}\right)$ was used to create a local infection rate of $100 \%$ with low morbidity. $S$ aureus were produced in the blood cultures of all infected cases. Thus, we created an infection model clinically similar to a patient with osteomyelitis and sepsis who underwent intramedullary nailing.

Retaining an implant in the presence of an underlying infection without antibiotics treatment leads to weaker callus and impedes callus maturation compared with noninfected controls in a rat model. A future study like the current study can be repeated to see whether antibiotic treatment would make a difference. This model permits setting the stage for further investigations to study the influence of different interventions (hardware present versus absent, antibiotherapy present versus absent) on fracture healing in implant-associated osteomyelitis. Our findings also suggest biopsy studies of patients with infected nonunions with hardware present versus absent using an observational design. 
Acknowledgments We thank Neslihan Onder MD, for her assistance of microbiological assessment and Kamber Kasali for his statistical analysis.

\section{References}

1. Berkes M, Obremskey WT, Scannell B, Ellington JK, Hymes RA, Bosse M, Southeast Fracture Consortium. Maintenance of hardware after early postoperative infection following fracture internal fixation. J Bone Joint Surg Am. 2010;92:823-828.

2. Chen X, Schmidt AH, Mahjouri S, Polly DW, Jr., Lew WD. Union of a chronically infected internally stabilized segmental defect in the rat femur after débridement and application of rhBMP-2 and systemic antibiotic. J Orthop Trauma. 2007;21:693-700.

3. Chen X, Tsukayama DT, Kidder LS, Bourgeault CA, Schmidt $\mathrm{AH}$, Lew WD. Characterization of a chronic infection in an internally-stabilized segmental defect in the rat femur. $J$ Orthop Res. 2005;23:816-823.

4. Cheung KM, Kaluarachi K, Andrew G, Lu W, Chan D, Cheah KS. An externally fixed femoral fracture model for mice. $J$ Orthop Res. 2003;21:685-690.

5. Court-Brown CM, Keating JF, McQueen MM. Infection after intramedullary nailing of the tibia. Incidence and protocol for management. J Bone Joint Surg Br. 1992;74:770-774.

6. Cremieux AC, Carbon C. Experimental models of bone and prosthetic joint infections. Clin Infect Dis. 1997;25:1295-1302.

7. Darouiche RO. Treatment of infections associated with surgical implants. N Engl J Med. 2004;350:1422-1429.

8. Einhorn TA, Lane JM, Burstein AH, Kopman CR, Vigorita VJ. The healing of segmental bone defects induced by demineralized bone matrix. A radiographic and biomechanical study. $J$ Bone Joint Surg Am. 1984;66:274-279.

9. Filion TM, Li X, Mason-Savas A, Kreider JM, Goldstein SA, Ayers DC, Song J. Elastomeric osteoconductive synthetic scaffolds with acquired osteoinductivity expedite the repair of critical femoral defects in rats. Tissue Eng A. 2011;17:503-511.

10. Gristina A. Biomaterial-centered infection: microbial adhesion versus tissue integration. 1987. Clin Orthop Relat Res. 2004;427:4-12.

11. Histing T, Garcia P, Holstein JH, Klein M, Matthys R, Nuetzi R, Steck R, Laschke MW, Wehner T, Bindl R, Recknagel S, Stuermer EK, Vollmar B, Wildemann B, Lienau J, Willie B, Peters A, Ignatius A, Pohlemann T, Claes L, Menger MD. Small animal bone healing models: standards, tips, and pitfalls results of a consensus meeting. Bone. 2011;49:591-599.

12. Huo MH, Troiano NW, Pelker RR, Gundberg CM, Friedlaender GE. The influence of ibuprofen on fracture repair: biomechanical, biochemical, histologic, and histomorphometric parameters in rats. J Orthop Res. 1991;9:383-390.
13. Kalteis T, Beckmann J, Schroder HJ, Handel M, Grifka J, Lehn N, Lerch K. Moxifloxacin superior to vancomycin for treatment of bone infections-a study in rats. Acta Orthop. 2006;77:315319.

14. Lane JM, Sandhu HS. Current approaches to experimental bone grafting. Orthop Clin North Am. 1987;18:213-225.

15. Lucke M, Schmidmaier G, Sadoni S, Wildemann B, Schiller R, Stemberger A, Haas NP, Raschke M. A new model of implantrelated osteomyelitis in rats. J Biomed Mater Res B Appl Biomater. 2003;67:593-602.

16. Makridis KG, Tosounidis T, Giannoudis PV. Management of infection after intramedullary nailing of long bone fractures: treatment protocols and outcomes. Open Orthop J. 2013;7:219226.

17. Mehta M, Schell H, Schwarz C, Peters A, Schmidt-Bleek K, Ellinghaus A, Bail HJ, Duda GN, Lienau J. A 5-mm femoral defect in female but not in male rats leads to a reproducible atrophic non-union. Arch Orthop Trauma Surg. 2011;131:121129.

18. Melcher GA, Hauke C, Metzdorf A, Perren SM, Printzen G, Schlegel U, Ziegler WJ. Infection after intramedullary nailing: an experimental investigation on rabbits. Injury. 1996;27(Suppl 3):SC23-26.

19. Merritt K, Dowd JD. Role of internal fixation in infection of open fractures: studies with Staphylococcus aureus and Proteus mirabilis. J Orthop Res. 1987;5:23-28.

20. Moussa FW, Anglen JO, Gehrke JC, Christensen G, Simpson WA. The significance of positive cultures from orthopedic fixation devices in the absence of clinical infection. Am J Orthop. 1997;26:617-620.

21. Petty W, Spanier S, Shuster JJ, Silverthorne C. The influence of skeletal implants on incidence of infection. Experiments in a canine model. J Bone Joint Surg Am. 1985;67:1236-1244.

22. Reikeras O, Reigstad A. Healing of stable and unstable osteotomies in rats. Arch Orthop Trauma Surg. 1985;104:161-163.

23. Rightmire E, Zurakowski D, Vrahas M. Acute infections after fracture repair: management with hardware in place. Clin Orthop Relat Res. 2008;466:466-472.

24. Robinson DA, Bechtold JE, Carlson CS, Evans RB, Conzemius MG. Development of a fracture osteomyelitis model in the rat femur. J Orthop Res. 2011;29:131-137.

25. Schmidt AH, Swiontkowski MF. Pathophysiology of infections after internal fixation of fractures. J Am Acad Orthop Surg. 2000;8:285-291.

26. Waldvogel FA, Medoff G, Swartz MN. Osteomyelitis: a review of clinical features, therapeutic considerations and unusual aspects. 3. Osteomyelitis associated with vascular insufficiency. N Engl J Med. 1970;282:316-322.

27. Worlock P, Slack R, Harvey L, Mawhinney R. The prevention of infection in open fractures: an experimental study of the effect of fracture stability. Injury. 1994;25:31-38. 